\title{
RHÉTORIQUE DE L'INGENIVM ET PERSONNALITÉ LITTÉRAIRE
}

\section{CRISTINA MÜLLER}

The purpose of this study is to briefly outline the historical evolution of the ingenium in the premodern culture. A rhetorical and literary concept, the ingenium organizes the ancient notion of the capacity of invention and of artistic inspiration, and eventually becomes a central concept in the classic Aesthetic thought. But its meaning and evolution are also intimately related to the discovery of the individuality, and to the tension between natura (the natural, inborn characteristics) and ars, doctrina (acquired, secondary, elaborated characteristics of an individual style). From Plato and Aristote, through Cicero, Quintilian and Erasme, the classic Aesthetics articulates a question still meaningful for the modern reflection on the individual : how can the tension between the acknowledged diversity of ingenia and the necessity for a unifying discipline like Rhetorics be ultimately reduced.

\section{$L$ 'ingenium dans la culture ancienne}

À côté de la natura, l'ingenium possède, déjà en latin, des significations qui font de lui un synonyme de la nature individuelle, de la capacité d'invention et de l'inspiration artistique. C'est dans cet ancien concept, assez négligé par les philosophes, que les rhéteurs ont condensé leur réflexion sur l'individualité, et les artistes leur souci d'authenticité, bien avant qu'une pensée de la personnalité artistique puisse s'y esquisser.

L'histoire conceptuelle de l'ingenium est essentiellement liée à celle de la rhétorique latine. Cependant, la notion possède d'importantes racines dans la pensée grecque. Ainsi, même si l'ancien grec semble ne pas avoir un équivalent exact, à des époques différentes, deux hypothèses ont pu être 
formulées sur le mot grec d'origine. Ni $\mu \tilde{\eta} \tau \iota \varsigma$ (en vertu de sa signification d'intelligence pratique, nécessaire dans tous les arts) ${ }^{1}-$ ni $\alpha$ ’ $\gamma \chi^{i}$ ivor $\alpha$ (due à une composante intellectuelle commune $)^{2}$ ne saurait épuiser la sphère sémantique du mot latin, mais ils signalent les germes d'une conception sur la créativité qui s'organisera autour de celui-ci. L'ancien français en connaissait encore l'équivalent: c'est l'engin de Guillaume de Machaut, faculté de l'invention et de la création artistique, un élément fondamental dans cette image de l'écrivain qui commence à s'esquisser à l'époque ${ }^{3}$. L'ingenium s'octroie une place de choix dans les théories sur la créativité littéraire de la Renaissance, dans une république des lettres encore unitaire grâce à l'usage du latin. Le français moderne n'a pas retenu le terme dans cette acception, en lui préférant celui de genius 'génie'; cependant il admet l'adjectif 'ingénieux', à partir duquel il a dérivé le nom 'ingéniosité', synonyme d' 'habileté', 'astuce', 'subtilité'. L'italien et l'espagnol ont conservé le mot, y compris sa signification, en faisant de lui le concept nucléaire d'une théorie baroque qui représente une véritable tentative d'autonomie de l'esthétique en tant que discours théorique. Dans la modernité, le mouvement romantique dérivera de l'ingenium une catégorie fondatrice de son esthétique, le 'génie' au sens moderne de personnalité exceptionnelle, qui rend compte des

\footnotetext{
1 Mercedes Blanco, Les rhétoriques de la pointe. Baltasar Gracián et le conceptisme en Europe, Paris, 1993, p. 54.

2 Dans son Trattato dell'ingegno dell'huomo (Venezia, 1576), l'italien Antonio Persio proposait ce mot en tant qu'équivalent grec du latin ingenium. Zabarella, commentateur des Analytiques secondes d'Aristote, allait reprendre cette association, en découvrant sa définition

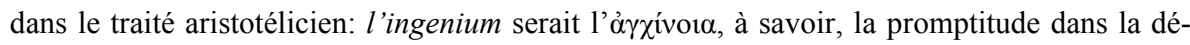
couverte du moyen terme d'un syllogisme. A partir de cette remarque d'Aristote, Avicenne avait développé la théorie de l'intellect possible. (Cf. H. Verin, La gloire des ingénieurs. L'intelligence technique du XVe au XVIII siècle, Paris, 1993, p. 18 sqq.)

3 On trouve le mot 'engin' au XIVe siècle au sens ancien, de grand esprit qui se définit par sa capacité d'invention (v. Jean de Meun in Guillaume de Lorris et Jean de Meun, Le Roman de la Rose, éd. F. Lecoy, Paris, Champion, 1965-1970, t.II, vv 16135-16147), et aussi au sens d'anagramme, d'écriture subtile, chez Guillaume de Machaut et d'autres écrivains médiévaux. (Jaqueline Cerquiglini, Un 'engin' si subtil, Guillaume de Machaut et l'écriture au XIVe siècle, Paris, Champion, 1985, pp. 213-214 et 245) Pourtant, le sens dominant que le mot semble avoir à l'époque médiévale es celui de ruse, habileté, ce qui explique peut-être la persistance de l'adjectif dans cette acception (Frédéric Godefroy, Dictionnaire de l'ancienne langue française et de tous ses dialectes du IX au XVe siècle, tome troisième, Paris, 1884, pp. 171-172)
} 
possibilités créatrices, de la fécondité de l'esprit humain. C'est aussi autour de ce mot qu'Érasme articule sa théorie du style et sa doctrine de l'imitation, en fournissant un de ces éléments essentiels qui articulent le passage de la pensée prémoderne à la modernité.

Le mot ingenium provient du latin in-gigno, dont le sens est 'inculquer dès la naissance'. Lorsque le mot s'applique à une chose, il en désigne les qualités naturelles (Virg., G. II 177). Appliqué à l'être humain, il possède tout d'abord un sens global : "les dispositions naturelles d'un être humain, tempérament, nature propre, caractère", selon un usage attesté chez plusieurs auteurs (P1., Trin. 667, Ter. Eun., 880, Ad. 71, Cic., Br. 204). Une deuxième signification isole les dispositions intellectuelles, l'intelligence (chez Cic. $B r$., 93), mais en essayant d'y inclure aussi la sensibilité (Tac., D. 21, Cic., Off. I 71). De cette acception, déjà le latin en a dérivé une autre, celle de 'dons naturels', pour dénoter le talent naturel, en tant que condition nécessaire de l'éloquence. La troisième signification applicable à un être humain semble s'infléchir déjà vers le contenu qui sera constitutif de la notion moderne : talent, génie en tant que faculté responsable de l'invention, de l'imagination ou même de l'inspiration (Cic., Pro Arch, 31, Tac., H. III 28, Sue., $A u, 89$ ).

L'ingenium en tant qu'élément naturel, inné, sur lequel prend appui l'art dans la formation individuelle, est présent, donc, dès très tôt dans la pensée rhétorique ancienne, mais sans posséder un profil bien défini. La multiplicité de significations présentes déjà en latin anticipe les divers développements que le concept connaîtra à l'époque prémoderne. Cependant, une signification plus constante, qui s'en dégage déjà en latin, renvoie à l'ensemble de données innées d'un individu nécessaires dans l'exercice d'une science, d'un art ou même de la philosophie. La solidarité entre l'individuel et l'ingenium semble donc être l'élément le plus constant que l'on puisse trouver dans son histoire ancienne.

\section{L'individuel dans la rhétorique ancienne}

La pensée de l'individuel n'est pas un thème privilégié par la pensée, philosophique ou rhétorique, de l'Antiquité ${ }^{4}$. La métaphysique de l'Un, avec

4 Pour un traitement du thème, infléchi sur celui de la réflexion sur l'artiste, on se 
sa méfiance envers la multiplicité des choses particulières, entraîne la dépréciation de l'individuel. En outre, quand l'individuel se dit en poète, il éveille des grands soupçons : c'est un individu d'une nature particulière, dont la singularité s'explique mal à partir du mouvement de spécification à l'intérieur du genre humain. La théorie de l'artiste inspiré chez Platon en témoigne. Pour Platon, le poète est un énergumène, un piètre instrument des dieux, tel le rhapsode Ion, qui se trouve complètement dépersonnalisé lorsqu'il interprète des poèmes devant son publique. L'autre individualité des disciplines de la parole, l'orateur, est réduite, avec le sophiste, à une figure de trompeur, dont l'art relève d'une fausse connaissance, d'une imitation vaine du philosophe. Même si, dans Phèdre, Platon semble admettre la nécessité de la rhétorique, son geste reste ironique.

Lorsque Aristote attribue une certaine supériorité au poète, il le fait en recourant à un principe de généralité, dont la poésie serait un lieu de représentation :

En effet, (l'historien et le poète) se distinguent (...) en ce que l'un raconte les événements qui sont arrivés, l'autre des événements qui pourraient arriver. Aussi la poésie est-elle plus philosophique et d'un caractère plus élevé que l'histoire; car la poésie raconte plutôt le général, l'histoire le particulier. Le général, c'est-à-dire que telle ou telle sorte d'homme dira ou fera telles ou telles choses vraisemblablement ou nécessairement ; c'est à cette représentation que vise la poésie, bien qu'elle attribue des noms aux personnages; le 'particulier', c'est ce qu'a fait Alcibiade ou ce qui lui est arrivé 5 .

Une relation entre la création littéraire et une faculté de création personnalisée, individualisée, n'est pas imaginable. La poésie est finalement acceptée dans la cité grâce à cette qualité philosophique relevant de l'éloignement du particulier, de l'individuel.

reportera à Jean Lecointe, L'idéal et la différence. La perception de la personnalité littéraire à la Renaissance, Genève, Droz, 1993, l‘Avant-propos', surtout p. 15 sqq. Pourtant, pour E. Zilsel (Le Génie, Paris, Minuit, 1993 [Die Entstehung des Geniebegriffes, Tübingen, 1926]), l'individualisme est déjà présent dans la culture grecque (v. surtout le chapitre 20, "La genèse de l'individualisme"), mais dans une forme que l'on pourrait qualifier de non réfléchie. C'est un manque que déploreraient, au XVI et au XVIIe siècle, Huarte de San Juan y, parmi les autres théoriciens du conceptisme, l'espagnol Baltasar Gracián, dans son Agudeza y arte de ingenio, où il souligne l'absence d'une réflexion et d'une théorisation adéquates de l'ingenio et de ses produits, les conceptos, pourtant tellement cultivés par les auteurs anciens.

5 Aristote, Poétique, éd. et trad. J. Hardy, Paris, 1990, 1451b. 
L'autre art qui s'était interrogé sur la nature de l'individuel et sur la créativité à travers le langage est la rhétorique, qui, en tant que discipline sophistique, était battue en brèche par Platon. Aristote réussit à l'infléchir dans une direction compatible avec la philosophie, en l'intégrant dans son encyclopédie. Pourtant, bien qu'elle soit considérée comme une "connaissance théorique et de causes", la rhétorique ne saurait être une science, car son objet n'est pas spéculatif, mais pragmatique. Sa position, reste, donc, inférieure par rapport aux sciences du sommet de la hiérarchie, telles la métaphysique ou la logique, et même la dialectique, science de la discussion, dont l'éloignement par rapport à la pensée abstraite est déjà évident. L'infériorité de la rhétorique découle de cet étroit rapport qui existe entre la réflexion du général et la valeur de la pensée: cette pensée est supérieure qui réussit mieux à rendre compte des aspects généraux de l'objet analysé. D'où l'obsession pour la catégorisation qui - à partir d'Aristote - va dominer l'histoire de la rhétorique, voire son conflit avec la philosophie, tout en témoignant de l'impossibilité radicale d'une saisie théorique de l'individuel. ${ }^{6}$

\section{L'ingenium dans la caractérisation de l'orateur}

La pensée rhétorique et poétique sera dominée pendant longtemps par cette exigence de généralité, qui place la figure de l'orateur et du poète sous le signe d'une représentation idéale. On retrouve cette image chez Cicéron, où elle est attribuée à Antoine, d'abord dans De l'Orateur et, dix ans après, dans L'Orateur. Grand orateur de la génération antérieure à Cicéron, Antoine ne croit pas qu'il y ait un homme vraiment éloquent (c'est-à-dire, une éloquence parfaite, idéale), en se contentant d'une image plus modeste, celle $\mathrm{du}$ disertus - l'orateur capable de se montrer persuasif dans ses discours ordinaires. Il en résulte que pour Antoine, l'orateur parfait (eloquens) n' existe pas en réalité: il est une idée ${ }^{7}$. C'est à cette idée que Cicéron va se

6 Selon Gilles Gaston Granger dans son Essai d'une Philosophie du style (Paris, 1968), l'esthétique est l'une des tentatives de dépasser cette impossibilité, ce qui explique la place qu'elle donne à l'individuel, et la solidarité historique entre l'émergence de la sensibilité et l'apparition d'une réflexion de la personnalité - principalement littéraire, mai aussi artistique, en général, au XVIe et XVIIe siècle).

7 'C'est qu'il y avait dans son esprit une idée de l'éloquence (species eloquentiae) que son intelligence discernait, mais qu'il ne voyait pas dans la réalité. (...)"(L'Orateur. Du meilleur genre d'orateurs, texte établi et traduit par Albert Yon, Paris, Belles Lettres, 1964, 
confronter dans sa tentative d'en esquisser le portrait :

Mettons-nous donc, Brutus, si nous en sommes capables, en quête de cet orateur qu'Antoine n'a jamais vu ou qui n'a jamais existé. Si nous ne pouvons pas en donner une image ressemblante, puisque Antoine disait aussi que c'était à peine au pouvoir d'un dieu, peut-être du moins pourrons-nous dire ce qu'il devrait être ${ }^{8}$.

Aux yeux d'Antoine, l'éloquence parfaite était à peu près incompatible avec la condition humaine, ce qui rendait difficile la tentative d'en trouver une définition. En revanche, il est significatif que Cicéron commence sa quête de l'orateur idéal par un geste complètement opposé : une description des trois styles qui prend comme point de départ la réalité variée des orateurs. L'image de l'eloquens n'apparaît pas comme développement d'une idée abstraite : c'est à travers des cas concrets que ses caractéristiques s'y dégagent. De plus, la relation qui existe entre la variété des éléments individuels et la division des styles, entre la perfection particulière d'un orateur et les exigences de perfection d'un genre, semble infléchir la représentation de l'orateur idéal vers des hypostases plus vraisemblables :

Il y a trois genres de style dans lesquels séparément certains ont excellé, mais (...) fort peu en tous également ${ }^{9}$.

Il n'y a pas d'orateur qui puisse maitriser également les trois styles : la nature individuelle pose une limite préalable à la compétence technique. Il suit que chaque orateur doit opérer un choix parmi les styles, en suivant sa nature, car, selon Cicéron, ceux qui ont joui d'une grande renommée se sont manifestés à l'intérieur de ce système. Le système hiérarchique des trois styles ${ }^{10}$ se constitue alors comme une limite indépassable dans la formation

V, 18.). Pourtant, dans De l'Orateur (texte établi et traduit par Edmond Courbaud, Paris, Belles Lettres, 1967), le même personnage place l'existence de l'orateur idéal sous le signe du possible, ou même du réel : "Mais cet orateur, il existe, à mon sens, et c'est Crassus que voici ; ou bien ce sera quelque autre qui, doué d'un génie égal au sien, mais ayant entendu, lu, écrit, davantage, complétera par ce quelque chose de plus le peu qui lui manque encore." (I, 95)

8 "Inuestigemus hunc igitur, Brute, si possumus, quem numquam uidit Antonius aut qui omnino nullus umquam fuit ; quem si imitari atque exprimere non possumus, quod idem ille uix deo concessum esse dicebat, at qualis esse debeat poterimus fortasse dicere." $\left(L^{\prime} O, \mathrm{~V}, 19\right)$

9 Cicéron, Orator, V, 20-21 : "Tria sunt omnino genera dicendi, quibus in singulis quidam floruerunt, peraeque autem (...) perpauci in omnibus."

10 La tripartition est la division des styles la plus répandue, face aux groupements septénaires (Hermogène) ou quaternaires (Macrobe et les poètes s'inspirant dans sa doctrine). Le 
individuelle. La première condition qui se dégage dans la quête du meilleur orateur est de 'répondre à l'un des trois styles'. L'individualité se heurte ici à sa première limite.

Mais on voit que dans la description d'un style, notamment du style grandiloquent ${ }^{11}$, il alterne des exigences générales (l'exigence de la gravité de la pensée, par exemple, qui porte sur la vaste catégorie des pensées aptes à être formulées dans ce style) avec des exigences plus strictement définies qui précisent la condition lexicale et syntactique du discours, telles la variété et l'abondance ou les deux caractérisations extrêmes de la phrase : inachevée, hirsute et lisse, construite. De cette manière, le système des styles permet que la variété des ingenia retrouve une certaine liberté. La réalisation individuelle d'un style admet des formes stylistiques éloignées, voire opposés, en faisant place à la différence. En outre, les trois styles ne sont pas égaux, car le style grave est supérieur dans une manière significative : il possède des qualités spécifiques, mais tout en opérant une sorte de récapitulation de la classification hiérarchique des styles ${ }^{12}$.

Il y a, donc, une certaine variété dans la réalisation individuelle d'un style, à laquelle s'ajoute la variété récapitulative du style grave, qui dépasse la linéarité stricte de la hiérarchie. On voit s'y esquisser une ligne de fuite à partir de la notion de variété, ce qui permet qu'une pensée de l'individuel soit mise en forme. Cette ligne de fuite prend appui sur la natura. La notion n'était pas nouvelle, car il s'agissait d'un type humain déjà présent chez Platon et Isocrate, dont l'excellence se fondait sur un don naturel, développé

\footnotetext{
système ternaire des styles (styli), genres (genera dicendi) ou caractères semble apparaître après Aristote, cependant sa paternité (attribuée à Théophraste ou à Démétrius de Phalère) n'est pas certaine. À la Renaissance, le système ternaire occupe aussi une place de choix dans les commentaires : «la division de loin la plus fréquente, celle dont on se sert pour qualifier l'aspect général du style d'un auteur, et surtout le ton d'un passage, es la division ternaire». - conséquence, peut-être, du fait qu'il «est plus strictement hiérarchisé» (J. Lecointe, p. 138. Voir aussi tout le chapitre «Le système hiérarchique du style», pp. 136 sqq. et 104-105)

${ }^{11}$ «En effet il y a eu des gens au style, si je puis dire, grandiloquent, avec une profonde gravité de pensée et majesté d'expression, véhéments, variés, abondants, graves, bien fournis et pourvus pour émouvoir et retourner les cœurs ; et cela, les uns avec une phrase rude, sévère, hirsute, imparfaite et inachevée, les autres une phrase lisse, construite, terminée». (L'O. V 20).

12 Alain Michel, Rhétorique et philosophie chez Cicéron. Essai sur les fondements philosophiques de l'art de persuader, Paris, 1960, p. 378, et J. Lecointe, p. 145 sqq.
} 
à travers l'étude et l'exercice ${ }^{13}$. C'est la notion dans laquelle la conception sur l'orateur de Crassus et celle d'Antoine coïncident, au-delà des différences qui les opposent:

Eh bien! continua Crassus, nature et génie, voilà suivant moi ce qui nous forme d'abord et le mieux à l'éloquence; et quant aux gens dont parlait Antoine, à ces faiseurs de traités, ce ne sont ni les règles ni la méthode, c'est la nature qui leur a manqué. Les dons d'intelligence et d'imagination sont essentiels, j'entends la facilité à recevoir des impressions vives, d'où résulte la finesse pénétrante de l'invention, l'abondance du développement, la fermeté et la solidité de la mémoire. Quelqu'un se figure-t-il qu'il atteindra ces résultats par le secours de l'art: il se trompe (ce serait trop beau, en effet, que l'art pût allumer l'étincelle ou provoquer l'éveil du génie; non, les aptitudes ne se greffent pas, ne s'octroient pas ainsi du dehors; elles sont en nous un présent de la nature.). Mais admettons; que dire alors des avantages suivants qui, ceux-là, sont sûrement apportés par l'homme à la naissance: langue agile, voix juste et sonore, poumons vigoureux, constitution robuste, visage agréable, belle prestance ? ${ }^{14}$

Nature et génie (naturam primum atque ingenium) dont on voit l'éloge cicéronien fait par Crassus, ne sont pas synonymes. Comme Quintilien plus tard, Cicéron entend intégrer dans la natura de l'orateur, à côté de l'ingenium, d'autres qualités qui relèvent de l'aspect, de la voix. Cependant, l'essentiel réside dans les 'dons d'intelligence et d'imagination' - c'est-à-dire, dans les capacités (motus) intellectuelles, spirituelles de l'individu, dans leur fonctionnement rhétorique, c'est-à-dire, général en ce qui concerne l'usage de la parole ${ }^{15}$.

13 Platon, Phèdre, 269d, Isocrate, Antidosis, 187. (Alain Michel, Rhétorique et Philosophie, p. 103 sqq.)

14 «Sic igitur, inquit, sentio, Crasssus, naturam primum atque ingenium ad dicendum uim adferre maximam; neque uero istis, de quibus paulo ante dixit Antonius, scriptoribus artis rationem dicendi et uiam, sed naturam defuisse. Nam et animi atque ingeni celeres quidam motus esse debent, qui et ad excogitandum acuti et ad explicandum ornandumque sint uberes et ad memoriam firmi atque diuturni; et si quis est, qui haec putet arte accipi posse - quod falsum est; praeclare enim res se habeat, si haec accendi aut commoueri arte possint; inseri quidem et donari ab arte non possunt; omnia sunt enim dona naturae, - quid de illis dicam, quae certe cum ipso homine nascuntur: linguae solutio, uocis sonus, latera, uires, conformatio quaedam et figura totius oris et corporis ? » (De l'Or., I 25.113-114)

15 L'ingenium est une notion privilégiée de la rhétorique, qui est la discipline responsable de l'éloquence et qui devient à partir de Cicéron "le plus achevé des arts". Jusqu'à Horace, en effet, la poétique même sera considérée comme une partie de la rhétorique et jusqu'au XIXe les diverses théories littéraires sur le style seront aussi élaborées à partir de 
Tout le développement ultérieur de la notion semble être contenu dans ce fragment: Cicéron invoque l'esprit (anima), dont relève les qualités intellectuelles, à côté du talent (ingenium) spécifique d'un orateur. Les qualités participant de l'ordre spirituel de la natura sont exprimées par des adjectifs dans le texte latin, mais ils rappèlent des concepts fondamentaux de la rhétorique: inuentio, elocutio, copia. La première qualité de l'esprit et du talent dans la caractérisation de l'eloquens est l'acuité ("la finesse pénétrante de l'invention"), déjà théorisée par Aristote dans l'Organon. Analytica Posteriora (I 34) en tant que rapidité de l'intelligence dans la découverte du terme moyen dans un syllogisme. Cette possibilité d'en faire un concept esthétique, déjà esquissée chez Cicéron, allait être exploitée au XVIIe siècle: sous le nom d'agudeza de ingenio ou d'acutezza degl'ingegno, elle fournira un concept essentiel à la poétique de l'ingenio à l'âge baroque. Les autres qualités requises par Cicéron sont l'abondance du développement et de l'élocution (essentielle pour la caractérisation du style élevé, style de l'auteur récapitulatif) et une mémoire solide et durable. Il s'agit d'une caractérisation plutôt qu'une vraie définition de l'ingenium, qui s'esquisse dans l'esprit de la rhétorique. Mais le germe de l'esthétisation du concept semble être déjà présent, car l'auteur y associe étroitement une qualité spécifique de l'individuel à la pratique artistique de la langue.

Crassus (le porte-parole de Cicéron dans ce dialogue) établit d'une manière tranchante la primauté de la nature et de l'ingenium, sans lesquels le processus de formation d'un orateur ne peut pas s'amorcer ${ }^{16}$. Mais, s'il est vrai que les dons spirituels s'octroient une place de choix, il faut aussi reconnaitre que, pour assurer la formation d'un orateur, l'esprit est insuffisant. Dans le reste du fragment on voit que le génie, pour aussi remarquable qu'il soit, ne peut pas compenser les défauts relevant de l'aspect ou de la voix.

l'art oratoire (Cfr. Jacqueline Dangel, "Imitation créatrice et style chez les latins" in G. Molinié et P. Cahné, Qu'est-ce que le style ?, Paris, 1994, p. 94.) Pour le thème du rapprochement entre poétique et rhétorique, on se reportera aussi à A. Michel, Rhétorique et Philosophie chez Cicéron, p. 97 sqq., surtout p. 100.

16 On trouve dans le discours de Crassus sur l'orateur une autre réminiscence de la conception surnaturelle du poète, qui justifie l'exaltation du talent individuel : "D'autres, en revanche, sont pourvus de ces qualités physiques et comblés des faveurs de la fortune, au point qu'ils ne semblent pas être nés comme le vulgaire, mais avoir été façonnés par la main d'un dieu." (De l'Or, XXIV, 115) 
Paradoxalement, l'ingenium et l'esprit sont ainsi relégués à un second plan. De plus, non seulement le manque, mais aussi la surabondance, l'excès de cette natura que Crassus exalte, éveille la méfiance du philosophe: De l'Orateur met en exergue le danger ${ }^{17}$ à propos du style de Gorgias. Tant représentatif de la civilisation grecque, qui est une civilisation des arts, le style sophistique de celui-ci aurait abouti à une virtuosité gratuite, qui ne saurait être le but de l'art oratoire ou de la poésie. L'art de l'orateur doit épouser la civilisation des mœurs, il faut que l'orateur rejoigne le philosophe ${ }^{18}$. Dix ans plus tard, dans L'Orateur, dans la description du grand style, cette attitude est encore présente. Style de la puissance, de la gravité, qui l'emporte sur tout autre type d'éloquence, le grand style s'inscrit entre deux qualifications extrêmes, l'excellence et l'excès :

Mais celui-ci que nous plaçons au premier rang, notre orateur grave, impétueux, ardent, s'il n'est né que pour ce genre sans tempérer son abondance par le mélange des deux autres, il est tout à fait à rejeter. L'orateur simple, parce qu'il parle avec finesse et a du métier, est tenu généralement pour sage ; le moyen, pour agréable; mais celui-ci, le très abondant, s'il n'en est rien d'autre c'est à peine qu'il passe pour sain d'esprit. (...) [il] a l'air d'un fou devant des personnes sensées et comme d'un homme ivre déchaîné au milieu de gens qui n'ont pas bu ${ }^{19}$.

On retrouve l'image du poète inspiré de Platon, présentée sous un angle assez proche de sa condamnation originaire. La description disqualifiante de

17 De l'Or. I 22.101 sq. ; 47.102 sq. ; II 18.75.

18 De l'Or. III 16. 61. Contre le geste de Socrate et des sophistes, qui ont séparé parole et pensée, Cicéron revient à leur ancienne unité (De l'Or. III 12). Mais son geste est analogue celui par lequel Aristote avait donné la dignité à la poésie: en le rapportant au général. Si traditionnellement la rhétorique était une science du particulier, et par cela considérée inférieure, elle doit récupérer justement cette capacité de généralisation : le cas particulier sera reporté à l'idée générale, en dégageant l'essence des circonstances, du contingent. Ce geste transforme l'orateur en philosophe. (Alain Michel, Rhétorique et Philosophie chez Cicéron, p. 5.) Il s'agit d'un thème récurrent dans l'histoire de la rhétorique, voire de son conflit avec la philosophie, réactualisé à la Renaissance, surtout après de Ciceronianus d'Érasme, où la philosophie que la rhétorique doit retrouver est la philosophia Christi, la spiritualité chrétienne.

19 «At uero hic noster, quem principem ponimus, grauis, acer, ardens, si ad hoc unum est natus aut in hoc solo se exercuit aut huic generi studuit uni nec suam copiam cum illis duobus generibus temperauit, maxime est contemnendus. Ille enim summissus, quod acute et ueteratorie dicit, sapiens iam, medius suauis, hic autem copiossissimus, si nihil est aliud, uix satis sanus uideri solet. (...) ... furere apud sanos et quasi inter sobrios bacchari uinulentus uidetur». (Or. XXVIII 99). 
Caius Fimbria dans Brutus est une application fidèle de ce critère, tout en signalant aussi l'idée d'un hors du système qui lui correspondrait. En s'interrogeant sur la raison pour la foule d'applaudir un tel orateur, Cicéron montre la place que la pensée ancienne a assignée à la nature excessivement douée, à la personnalité exceptionnelle, guettée par la démesure et le déséquilibre :

Cet homme qui ne disait rien sans crier et qui parlait une assez bonne langue, mais avec une sorte d'impétuosité torrentielle, se livrait à de telles fureurs, qu'on se demandait avec étonnement à quoi pouvait bien penser le peuple pour laisser à un pareil forcené une place parmi les orateurs ${ }^{20}$.

Dans une rhétorique qui s'allie à la philosophie, cet excès est sanctionné et la possibilité de sortir du système n'est pas du tout investie de sens et de valeur. Mais dans Pro Archia l'auteur revient aux idées platoniciennes de l'Ion, et aux Pythagoriciens, cette fois-ci en accordant le thème de l'inspiration irrationnelle à l'ensemble de la culture littéraire. ${ }^{21} \mathrm{C}$ 'est dans ce cadre que la nature s'émancipe de sa dépendance envers la doctrina, pour rendre possible la reconnaissance de son entière supériorité. Il s'agit tout d'abord des hommes exceptionnels, dont la force morale et la vertu sont presque divines, et qui peuvent briller sans l'aide de la science, mais dont la nature n'aboutit à sa perfection qu'à travers la discipline et le façonnement assurés par la science ${ }^{22}$. Au contraire, l'excellence du poète est différente, car elle découle de la seule force de sa pensée :

Il est d'ailleurs une notion que nous tenons des hommes les plus éminents et les plus instruits, c'est que, si toutes les autres études reposent sur un enseignement, des leçons, un art, le poète, lui, vaut par sa seule nature, ce sont les forces de sa pensée qui lui donnent l'essor, une sorte de souffle divin l'inspire. Aussi a-t-il pleinement raison notre illustre Ennius, quand il applique aux poètes l'épithète de "sacrés", voulant dire qu'ils apparaissent comme des êtres qui nous ont été confiés en quelque sorte par un don, par une faveur des dieux ${ }^{23}$.

20 Brutus, texte établi et traduit par Jules Martha, Paris, 1966, LXV - 233, p. 84 : "qui omnia magna uoce dicens, uerborum sane bonorum cursu quodam incitato, ita furebat tamen, ut mirarere tam alias res agere populum, ut esset insano inter disertos locus."

${ }^{21}$ Alain Michel, «La rhétorique, sa vocation et ses problèmes: sources antiques et médiévales» in Marc Fumaroli, Histoire de la Rhétorique dans l'Europe moderne. 1450-1950, p. 27.

22 Pour le poète Archias 7.15, in Cicéron, Discours, tome XII, texte établi et traduit par Félix Gaffiot, Paris, 1989.

23 Idem, 8.18: Atque sic a summis hominibus eruditissimisque accepimus, ceterarum re- 
La poésie se développe donc en tant qu'exception: si dans l'art oratoire la personnalité exceptionnelle est difficile à intégrer, elle trouve sa place dans la création poétique, comme une reconnaissance de l'excellence individuelle. La personnalité excessivement douée ne saurait pas trouver sa place dans une activité publique et quotidienne comme l'art oratoire, mais elle est intégrée dans l'ensemble de la culture par le biais d'une activité "sacrée": la poésie. Le système clos de la rhétorique est dépassé et la poésie redevient un lieu de contact avec le sacré, comme il l'avait été dans les représentations religieuses anciennes du poète.

Chez Quintilien, la primauté de la natura est aussi affirmée sans ambages dans plusieurs passages ${ }^{24}$. La natura est en premier lieu l'ingenium de l'orateur, cet ingenium qui a partie liée avec le sacré à travers l'animus, qui est d'origine divine ${ }^{25}$. Mais il relève aussi d'une qualité au plus haut degré individuelle, car la faculté intellectuelle n'est pas identique chez tous les hommes. La visée pédagogique de son traité s'appuie sur une distinction nette entre la différence qualitative des ingenia individuels: la connaissance et reconnaissance de l'individualité unique constitue le point de départ de toute formation individuelle. ${ }^{26}$

Mais dans ses Institutions Oratoires Quintilien doit aussi prendre parti contre l'idée d'une autonomie totale de la natura, idée qu'il trouve chez des orateurs contemporains, des praticiens doués d'une formation élémentaire qui contestent l'utilité de la formation technique dans la pratique de la parole $^{27}$. Le fait est significatif, car il met en exergue l'origine de l'attitude contradictoire envers la natura, attitude qui semble dominer la tradition théorique ancienne: si l'on reconnaît sa primauté, voire la supériorité par rapport à l'art, il en reste néanmoins une méfiance, une réserve, car la nature tente de franchir la tutelle de l'art. Cette attitude se manifeste dans la critique de la natura excessive, dont Quintilien révèle à son tour la présence dès l'enfance:

rum studia et doctrina et praeceptis et arte constare, poetam natura ipsa ualere et mentis uiribus excitari et quasi diuino quodam spiritu inflari. Quare suo iure noster ille Ennius sanctos appellat poetas, quod quasi deorum aliquo dono atque munere commendati nobis esse uideantur.

${ }^{24}$ Inst.or., I, pr., 26-27 ; III 2.1 ; III 3.2 ; III 5.1.

25 idem, I, 1,1 ; XII 2.28.

26 ibidem, II 8.1.

27 ibid., II 11-12 et 17-19. 
la facilité qu'un élève manifeste dans l'apprentissage est trompeuse, car elle n'implique pas la profondeur de l'acquis ${ }^{28}$.

L'opposition natura/ars y est donc élaborée selon une solution éclectique que l'on trouvait déjà chez Cicéron: "il faut l'union des deux pour faire un orateur consommé" ${ }^{29}$. Mais le rôle de l'ars semble déjà plus marqué par rapport à la place que Crassus (porte-parole de Cicéron) lui assignait: dans cette alliance, le dosage est très important, car il diffère selon le style cultivé:

Car si l'on sépare entièrement la nature et l'enseignement, les dons naturels pourront beaucoup, même sans l'enseignement, mais celui-ci ne pourra rien sans la nature. $\mathrm{Si}$, au contraire, il y a coexistence en proportions égales, les dons naturels auront alors, je crois, la prépondérance chez l'orateur moyen, mais l'orateur consommé devra plus, à mon sens, à l'apport de l'enseignement qu'à la nature ${ }^{30}$.

Le rapport entre la nature et l'enseignement (et de l'art) n'est pas proportionnel dans l'acquisition de l'éloquence. Plus la place occupée par le style est supérieure, plus l'art devient important. Si la natura est essentielle comme point de départ dans la formation d'un orateur, l'importance qu'on lui assigne s'amoindrit au fur et à mesure que la qualité de l'orateur augmente. Le schéma proposé par Quintilien est donc éclectique, mais en accordant de plus en plus importance à l'ars, à l'enseignement. Il faut que l'orateur possède les dons de l'ingenium, de la natura, mais ces dons devraient être portés à un état parfait à travers l'ars et l'exercice. Cette relation s'articule dans un schéma hiérarchique, où l'ingenium et la nature occupent une position subordonnée. Comme nous fait remarquer Lecointe, c'est aussi Quintilien qui, dans la mouvance d'Aristote, élabore à nouveau l'opposition rhétorique natura/ars, en la rattachant à l'opposition matière/forme :

... les dons naturels sont le matériau par rapport à l'enseignement (natura materia doctrinae est); celui-ci modèle ; ceux-là sont modelés. L'art n'est rien sans la matière; la matière, même sans art, a du prix, mais la perfection de l'art l'emporte sur la matière la plus riche ${ }^{31}$.

28 Ibid., I 3.3-4.

29 Ibid., II.19.

30 "Nam si parti utrilibet omnino alteram detrahas, natura etiam sine doctrina multum ualebit, doctrina nulla esse sine natura poterit. Sin ex pari coeant, in mediocribus quidem utrisque maius adhuc naturae credam esse momentum, cosummatos autem plus doctrinae debere quam naturae putabo ..." (Inst.or. II 19.2)

31 "Denique natura materia doctrinae est : haec fingit, illa fingitur. Nihil ars sine materia, materiae etiam sine arte pretium est ; ars summa materia optima melior." (idem, II 
Cette solution éclectique à la dualité ars/ingenium va dominer par le biais du système pédagogique toute la tradition littéraire jusqu'à l'aube de l'époque moderne. L'apport horacien sera décisif dans cette histoire, où la dépréciation rhétorique de la nature excessive contaminera aussi l'image du poète inspiré. On voit une fois de plus l'intellectualisme de la culture ancienne s'orientant contre les éléments individuels trop marqués, réminiscences de la théorie traditionnelle de l'inspiration divine du poète. Dans ses grands moments, la rhétorique ancienne épouse la philosophie, en renfermant l'ingenium dans un système hiérarchique - celui des styles (au niveau individuel) ou celui des disciplines (au niveau de la culture). Comme témoigne la caractérisation du poète, l'individuel (pris dans sa manifestation d'ordre esthétique) apparaît généralement comme un phénomène non-hiérarchique, et, par conséquent, comme négation des structures, ce qui le rend théoriquement insaisissable pour la pensée ancienne.

Dans la tradition ancienne, la réflexion sur l'individualité s'esquissait autour de la notion de variété, solidaire avec les ingenia individuels. La tradition ultérieure, notamment à partir des commentaires macrobiens sur le style de Virgile, développera l'idée d'une variété en tant que marque de l'auteur récapitulatif, dont le style résume et contient les autres styles. ${ }^{32}$ Grâce à ce pouvoir de récapitulation, l'ingenium exceptionnel échappe à la condition commune: Macrobe l'identifie avec l'inspiration divine de formule platonicienne. C'est à partir du même thème de la variété que l'on évoluera vers la notion moderne du génie, lorsque l'ingenium exceptionnel arrivera à s'émanciper du système hiérarchique des styles ${ }^{33}$.

Tel est le processus que le Cicéronien ${ }^{34}$ met en évidence: c'est avec Érasme que s'opère le glissement de sens entre l'ancienne signification de l'ingenium et l'acception moderne, celle de génie. C'est aussi dans ce contexte que le geste polémique d'Érasme récupère sa pertinence majeure, et semble

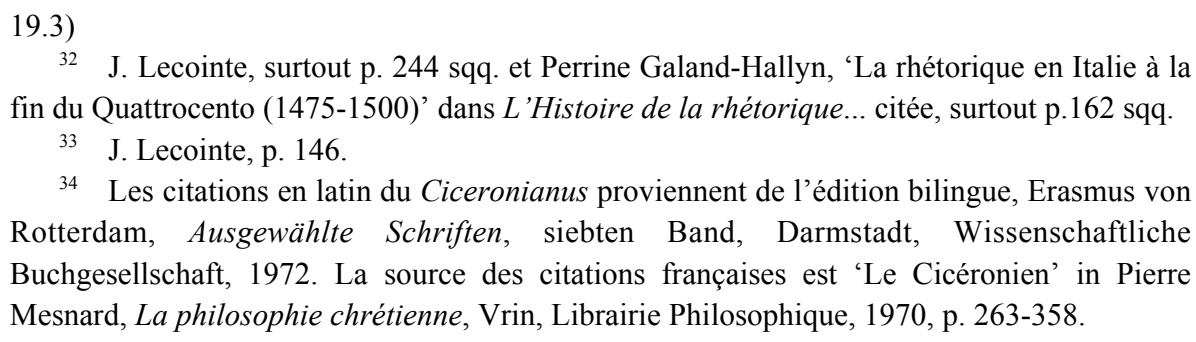

34 Les citations en latin du Ciceronianus proviennent de l'édition bilingue, Erasmus von Rotterdam, Ausgewählte Schriften, siebten Band, Darmstadt, Wissenschaftliche Buchgesellschaft, 1972. La source des citations françaises est 'Le Cicéronien' in Pierre Mesnard, La philosophie chrétienne, Vrin, Librairie Philosophique, 1970, p. 263-358. 
dépasser les limites impliquées par sa poétique encore imitative.

Ciceronianus : imitation et liberté de l'ingenium

Avec le Ciceronianus, Érasme s'inscrit au cœur même du débat renaissant sur l'imitation, au cours duquel le concept d'ingenium commence à s'imposer sur ceux de la doctrina et de l'ars. Le dialogue erasmien marque un moment important dans la querelle des cicéroniens qui oppose plusieurs humanistes insignes, dans une polémique aux effets décisifs sur la constitution d'une nouvelle pratique littéraire, plus libre face à l'imitation.

Ciceronianus $^{35}$ met en exergue la composante individuelle de l'écriture: le geste créateur de l'écrivain surgit de l'individualité irréductible, de l'ingenium personnel. La nature individuelle prend le pas sur l'imitation servile des modèles anciens, notamment de Cicéron, idole unique des cicéroniens. Tout en s'inscrivant dans la tradition humaniste de l'imitatio, Érasme va en élaborer un concept plus libre : il s'agit pour l'homme de lettres d'abandonner la pratique reproductrice par laquelle on s'empare des idées et formules d'autres, pour trouver en soi-même les plus hautes qualités d'un style.

La pensée d'Érasme s'articule autour des notions relevant de l'univers culturel ancien, particulièrement de l'œuvre de Quintilien, qu'Érasme préfère à celle de Cicéron. Le concept d'imitation d'Érasme constitue - à peu d'exceptions près ${ }^{36}-$ la position la plus avancée que l'époque permet de prendre à cet égard. Il est significatif que l'auteur mette en œuvre, pour le

35 Le dialogue met en scène trois personnages qui débattent la conception de style des cicéroniens, présentée sous un angle ironique, voire caricatural. Bulephorus est le porteparole de l'auteur, et il s'octroie le rôle principal, son nom signifie 'celui qui porte conseil'. Nosoponus est un cicéronien possédé par le désir de se rassembler à son modèle jusqu'à l'identité ; ce désir se manifeste comme une authentique maladie amoureuse, comme nous font remarquer d'ailleurs les personnages mêmes. Hypologus a aussi un rôle important : il joue l'intermédiaire, en adoptant successivement des attitudes contraires, tout en faisant le jeu de Buléphore. Le but déclaré du dialogue est celui de guérir Nosoponus 'par le logos' (qui a non seulement le double sens de raison et parole, mais renvoie aussi au verbe divin, Jésus Christ, qui est présenté, à la fin du dialogue comme la réalité ultime que l'orateur exprime dans son discours).

36 L'Arétin, par exemple, dont l'attitude de contestation envers le cadre normative de la création este extrême : apparemment c'est le seul à affirmer la liberté totale de l'ingenium. (Zilsel, op.cit., p. 207 sqq. ) 
développer, l'ancienne métaphore de l'abeille, devenue notion centrale de la pensée humaniste après avoir été élaborée à nouveau par Pétrarque. ${ }^{37} \mathrm{La}$ pensée erasmienne s'articule dans ce cadre traditionnel, dont les éléments principaux sont encore conservés. Contre Cicéron et ses imitateurs, qui exigent l'enfermement de l'ingenium dans un seul modèle, Érasme préconise un éclectisme des sources, formulé dans une 'règle aux imitateurs' qu'il puise chez Quintilien. Il s'agit d'une règle qui aura appartenu à Zeuxis, célèbre auteur du portrait d'Hélène de Crotone, que Quintilien reprend, en exigeant à son tour :

de ne pas se contenter de lire un seul auteur, ni de les lire tous pêle-mêle, ni les premiers venus, mais parmi les plus célèbres de faire un choix des meilleurs. : c'est ainsi qu'il réserve à Cicéron la première place, mais non la seule." (p. 281 $)^{38}$

L'acquisition d'un style personnel, qui soit en même temps 'le meilleur', se réalise par une synthèse des traits stylistiques que chaque auteur trouve chez des modèles admirés, à la manière de Zeuxis, qui a réuni dans un seul portrait les traits les plus beaux des cinq filles, les filles les plus belles de la ville. Il s'agit d'un double choix, et d'un 'choix prudent' qui implique faire le tri non seulement parmi la foule des cultivateurs de la parole, mais aussi à l'intérieur d'un style personnel, pour en distinguer les traits les plus remarquables, pour "éviter certains erreurs" et en "corriger d'autres" (p. 315). C'est-à-dire, par une opération critique, par un acte de discernement et de jugement qui exprime d'une manière profonde la personne de l'écrivain. C'est dans l'examen critique des exemples d'œuvres et de styles que la personnalité littéraire se découvre et se manifeste soi-même, et que le style individuel aboutit à sa forme unique. ${ }^{39}$ La liberté de l'ingenium individuel s'affirme donc d'abord comme possibilité d'imiter plusieurs modèles, notamment dans le choix que chaque esprit doit opérer à l'intérieur de cette

37 J. Lecointe, p. 287.

38 "... nec unum esse legendum nec omnes nec quoslibet, sed ex praecipuis deligendos aliquot eximios, inter quos Ciceroni primas tribuit, non solitudinem." (p. 64)

39 On trouve ici l'origine renaissante du discreto, l'idéal humain du XVIIe siècle, chez qui le style devient un style de vie, fondé sur l'exercice constant du discernement. Le discreto, dont l'incarnation parfaite est Critilo, personnage central du roman El Criticón, de Baltasar Gracián, se choisit et se construit soi-même d'une manière similaire à celle que l'on suit pour construire un style chez Érasme. C'est-à-dire, à travers de (bons ou mauvais) choix qu'il doit opérer parmi des conduites exemplifiées par d'autres personnes. Le discernement, le jugement est ainsi proposé comme un élément de liaison entre éthique et esthétique. 
variété : ainsi les paroles "ne sont pas le signe de notre esclavage, mais de notre liberté" (p. 291).

Le style dépend donc de l'individualité de l'auteur, ce qui rend l'ingenium personnel inimitable. L'authenticité est l'effet non seulement des traits positifs d'un style, mais aussi des défauts que l'auteur n'a pas su éviter parfois ou qu'il a contrôlés autrefois grâce à un équilibre unique entre ses qualités et ses défauts. C'est pourquoi Érasme, en suivant en cela Quintilien, refuse l'imitation servile, en reconnaissant l'authenticité comme condition nécessaire de tout style personnel. La négation porte sur la possibilité même de réaliser une imitation fidèle : l'idée même se heurte à une impossibilité réelle d'imiter Cicéron, qui découle de la mauvaise connaissance de son œuvre, et aussi de l'inégalité de celle-ci, car l'auteur ne s'élève pas toujours à la hauteur de son idéal stylistique. À cette imitation extérieure manquerait inévitablement l'équilibre de l'original, le dosage des caractéristiques strictement personnalisées qui fondent le style individuel:

si nous en croyons Quintilien, elles [les vertus] sont inimitables et nous ne pourrions les acquérir en suivant son exemple et ses préceptes : il faudrait nous adresser directement à Minerve. Mais si ces dons nous font défaut, que devient l'imitation des qualités dont nous avons parlé ?" (p. 296) ${ }^{40}$.

Cette exigence d'authenticité devient essentielle pour Érasme : renoncer à cultiver sa propre nature c'est s'éloigner de son individualité, d'où l'idée que l'imitation a des effets aliénants : "si l'on s'efforce d'égaler Cicéron, l'on s'exposera à perdre son style" (p. 297). Le style et l'œuvre, deviennent le lieu de manifestation de la personnalité. Le profil chaque fois différent de l'ingenium se réfléchit dans un style chaque fois différent. La quête du vrai cicéronien est vouée à l'échec, car on ne saurait trouver Cicéron 'au complet' sinon en lui-même. ${ }^{41}$ Désormais le processus d'acquisition d'un style individuel sera au plus haut degré personnalisé, même s'il est encore confiné au cadre imitatif de la tradition. On voit le schéma de Quintilien se renverser: tandis que pour celui-ci l'orateur consommé devait plus à l'art qu'à son ingenium, Érasme pose l'irréductibilité des dons naturels en tant

40 “... [haec virtutes] si Fabio credimus, sunt inimitabile nec ab exemplo praeceptisve peti possunt, sed a Minerva. Hae vero si absint, qualis erit eorum quae commemoravimus imitatio ?" (p. 122) Érasme renvoie ici à Quintilien, Inst. or. X 2.24 sq.

${ }_{41}$ "Si tu veux exprimer Cicéron tout entier, tu ne peux donc t'exprimer toi-même ; et si tu ne t'exprimes toi-même, ton discours sera comme un faux miroir...” (p. 314) 
que limite absolue dans la formation individuelle. Une sorte de dialectique de l'individuel nous est ainsi proposée: la nature individuelle relève de l'ordre de l'universel, comme une sorte de "déterminisme universel", mais elle représente aussi "la puissance de l'individu", "le principe spécifique, le 'quid proprium' des êtres, le mouvement qui les porte vers telle ou telle fin, dans telle ou telle direction." 42

Cela implique aussi un renversement axiologique: la valeur d'un style ne réside pas dans sa similitude avec un modèle, car la variété individuelle, la différence, sont désormais investies elles-mêmes de sens et de valeur. C'est tel le sens de la métaphore des pierres précieuses qu'Érasme invoque à la fin du Ciceronianus: elle lui permet de dissocier, contre la conviction des cicéroniens, similitude et valeur, car la dissemblance n'empêche pas qu'une œuvre soit valeureuse (p. 313). D'ailleurs, la caractérisation de nombreux styles individuels que l'on trouve dans la seconde partie du dialogue renforce cette idée: là, valeur stylistique et proximité au modèle s'excluent réciproquement dans la plupart des cas. Voilà, par exemple, la difficulté exemplaire que le cas de Pétrarque pose à Nosopon :

Buléphore. - Le prince de cette éloquence renaissante semble avoir été François Pétrarque, qui fut très célèbre à son époque (...). Son génie était brillant, sa science très étendue et la force de son éloquence n'était pas médiocre.

Nosopon. - Je le reconnais. D'ailleurs il possède une connaissance de la langue latine telle que tu la souhaiterais (...) Mais comment pourrait-on le dire cicéronien alors qu'il n'a même pas ambitionné ce titre. (p. 328) ${ }^{43}$

Le cicéronien Nosopon applique un filtre sévère dans l'examen des hommes des lettres: une conception du style qui prend comme critère le modèle cicéronien, dans ses moindres détails formels, grammaticaux. ${ }^{44}$ Cependant, si

42 Jean-Claude Margolin, L'idée de nature dans la pensée d'Érasme, Basel und Stuttgart, Helbing \& Lichtenhahn, 1967, p. 16.

43 p. 238 pour le texte latin.

44 Nosopon a fait trois répertoires de l'œuvre cicéronienne : deux alphabétiques, contenant l'un, un lexique et une concordance, et l'autre, les tropes, les figures contenus dans l'œuvre cicéronienne. Son adhésion au langage cicéronien est extrême : il essaye d'utiliser pour écrire soi-même seulement les formes contenues dans son premier répertoire : comme, par exemple, amo, amas, amat, mais pas amamus et amatis (peu ou nullement présentes chez Cicéron) ; amabam, mais pas amabatis ou bien amor, amoris, amori et amorem, mais pas $\hat{o}$ amor, hos amores, horum amorum etc. (p.269). Son attitude découle d'une confusion, entre “corpus et langue réellement parlée, anatomie et être vivant, observation fait de l'extérieur et 
le critère d'évaluation d'un auteur n'est pas l'excellence du style en tant qu'individuel, alors toute l'histoire de l'écriture est nulle, car il n'y a pas un seul cicéronien (à savoir, une copie identique de Cicéron). L'absurdité de cette position met en évidence le conflit inévitable qui se prépare dans la culture imitative de la Renaissance tardive et qui mettra en cause le concept même d'imitation.

Mais, malgré son attitude, le jugement de Nosopon est très nuancé ; ce cicéronien ardent fait preuve d'une très bonne connaissance des lettres anciennes et contemporaines, et d'une sensibilité réelle envers la spécificité de chacun des auteurs convoqués. Cet examen est le signe le plus clair de l'irréductibilité et l'inimitabilité de l'ingenium, qui finit par s'imposer au 'malade' même. Car c'est, paradoxalement, Nosopon ${ }^{45}$ qui invoque le jugement de goût lorsqu'il reconnaît la valeur de Politien et affirme que :

par son esprit tout à fait angélique, [Politien] fut un rare prodige de la nature dans quelque genre littéraire qu'il abordât ; mais son style est sans aucun rapport avec celui de Cicéron : il faut y goûter des qualités toutes différentes." (p. 330 ${ }^{46}$

L'ironie socratique du modèle s'y fait sentir, car les affirmations de Nosopon impliquent des conséquences contradictoires par rapport à sa propre théorie du style. En invoquant un autre critère que la similitude au modèle, Nosopon affirme sans vouloir l'existence des qualités stylistiques

connaissance de l'intérieur, c'est-à-dire pratique effective de la langue qui est toujours à quelque degré capacité d'inventer des formes inédites ; car telle forme, absente du corpus cicéronien, peut cependant avoir appartenu à son latin, en ce sens qu'au besoin il l'aurait employé, voire inventé." (Jacques Chomarat, Grammaire et rhétorique chez Erasme, Paris, Belles Lettres, 1981, vol. II, p. 819) Chomarat nous fait remarquer aussi la relation qui existe entre l'attitude de Nosopon et la tentative échouée d'Érasme de récupérer le latin vivant des Anciens, en dépassant le latin momifié des scolastiques par un usage quotidien du latin adapté aux besoins modernes : il s'agit du latin composite, appris à travers la lecture de divers écrivains (idem, p. 821).

45 Lecointe fait remarquer aussi l'importance de la position de Nosopon pour l'amorce d'un discours sur l'authenticité de l'écrivain : “d'une certaine façon c'est Nosopon, le Cicéronien, qui ouvre la voie. Entouré de son arsenal des dictionnaires, de fiches, avec son souci de précision cicéronienne maniaque, Nosopon a relevé notamment 'les formulas loquendi nostro peculiares', les expressions typiques de Cicéron.” (p. 449).

46 "prorsus angelica fuisse mente, rarum naturae miraculum, ad quodcunque scripti genus applicaret animum, sed nihil ad phrasim Ciceronis ; diversis virtutibus suspiciendus est." (p.244) 
exceptionnelles relevant d'un critère complètement différent. Cela revient à dire que les qualités d'un style ne constituent plus l'objet d'une norme universelle, car même le cicéronien ardent qu'est Nosopon admet qu'Ange Politien est un ingenium divin, dont l'excellence relève d'autres vertus que celles de Cicéron. On voit s'imposer ici en raccourci l'idée d'une différence personnelle directement saisissable dans les traits stylistiques ; la pratique littéraire est un lieu privilégié où l'on peut saisir l'individualité. C'est dans ces implications ironiques de l'affirmation de Nosopon que se laisse préfigurer le thème moderne du goût esthétique. Le choix parmi les diverses caractéristiques stylistiques que la réalité littéraire offre à un moment donné ne s'appuie pas sur des concepts rationnels. Il y a à la fois l'absence de concept et l'impossibilité d'une explication rationnelle (Nosopon ne sait comment justifier l'attitude favorable à l'égard des non-cicéroniens), mais aussi la reconnaissance du plaisir esthétique que ces œuvres lui provoquent. Le plaisir esthétique s'enracine dans la nature individuelle (ce qui élimine la possibilité de le reporter à un concept), mais en même temps, il s'impose d'une manière universelle, ce dont semble témoigner l'attitude de Nosopon qui le reconnaît contre sa volonté et contre sa conception sur le style.

L'articulation du thème devient encore plus nette lorsque Nosopon, et en réplique Buléphore, invoquent la qualité insaisissable d'un style, en utilisant une expression cicéronienne (nescio quid) devenue célèbre avec Pétrarque : le non so ché nommant l'incapacité de l'artiste de conceptualiser la beauté. L'aconceptualisme du jugement de goût théorisé plus tard par Kant est une réalité que Nosopon, cible de l'ironie erasmienne, est obligé de dénoncer, tout en signalant qu'une nouvelle conception esthétique est mise en scène contre la représentation traditionnelle, quantitative et imitative, de la beauté artistique.

\section{Le style : individuation ou individualisation?}

Dans la conception extrême incarnée par Nosopon, il n'y a pas de place pour la liberté et l'expression de l'ingenium: la formation de l'homme de lettres et sa création doivent suivre de très près le modèle cicéronien, posé comme norme universelle du style. Cette perspective poussée à l'extrême par Érasme était pourtant celle d'un nombre important des humanistes de 
l'époque ${ }^{47}$. Dolet réplique dans son De imitatione ciceroniana $^{48}$, où il articule la position antithétique qu'était celle des cicéroniens : le style de Cicéron est paradigmatique pour les professionnels des ars dicendi, pour une partie de la nature humaine qui ne leur est pas spécifique (à savoir, propre à l'exclusion de tout autre profession), mais qui est virtuellement présente dans tout esprit individuel. ${ }^{49}$ L'imitation fidèle du style cicéronien n'est pas seulement possible, mais elle est aussi la seule manière pour l'homme de lettres d'aboutir à la perfection individuelle, car celui-ci incarne une idée générique de perfection. Pour les cicéroniens, l'ingenium était donc une donnée unificatrice du genre humain, et un facteur d'individuation à travers le style.

À cette Idée universelle incarnée par Cicéron, Érasme oppose le decorum peculiare, "le principe d'universelle relativité subjective" ${ }^{50}$. En partant du decorum personae, principe d'adaptation entre le discours et la personne dans la rhétorique ancienne, et du thème de la variété des natures individuelles, la conception sur le style d'Érasme s'appuie sur le libre choix des modèles, pour permettre l'épanouissement de l'ingenium personnel. Pour lui aussi, l'ingenium est tout d'abord un trait spécifique, présent chez tout individu humain :

Chacun des mortels a son génie particulier qui lui est inné. Ce dernier possède une telle force qu'une nature prédisposée à tel genre d'éloquence s'efforcera en vain d'en acquérir une autre. Car lutter contre les dieux n'a jamais profité à personne, comment disent les Grecs." (p. 312) ${ }^{51}$

Mais l'accent qu'Érasme met sur l'ingenium constitue déjà une première

${ }^{47}$ Dans ce dialogue, Érasme prend le contre-pied des positions théoriques opposées à sa propre doctrine, notamment des celles de Bembo. (Cfr. J. Lecointe, p. 430)

${ }_{48}$ Cfr. Terence Cave, The Cornucopian Text. Problems of writing in the French Renaissance, Oxford, Clarendon Press, 1979, p. 48 sqq.

49 "ars tota dicendi, imago quaedam est et similitudo in ingeniis omnium insita" (De imitatione ciceroniana, in L'Erasmianus sive Ciceronianus d'Étienne Dolet, 1533, (facsimile), ed. E.V. Telle, Genève, 1974, p. 163, cité par T. Cave, p. 51.

${ }^{50}$ J. Lecointe, p. 432. L'idée d'un decorum peculiare qui constitue l'axe de l'inflexion 'personnaliste' présente dans la pensée érasmienne de l'individualité est le vrai fil conducteur de cette étude que Lecointe écrit sur la personnalité littéraire à la Renaissance.

51 "Habent singula mortalium ingenia suum quiddam ac genuinum, quae res tantam habet vim, ut ac hoc aut illud dicendi genus natura compositus frustra nitatur ad diversum. Nulli enim bene cessit $\theta \varepsilon$ co $\alpha$ í $\alpha$ quemadmodum Graeci solent dicere.” (p. 178) 
différence qui l'oppose aux cicéroniens: il s'agit d'une composante différentielle de l'individualité humaine, une donnée infaillible, que le travail, l'exercice ou l'érudition ne sauraient donner, mais seulement respecter et développer. En citant un proverbe grecque, ${ }^{52}$ Érasme fait apparaître, en dehors de l'innéisme rationaliste qui définit sa doctrine du style, l'élément surnaturel que la tradition assignait à l'inspiration : l'ingenium a partie liée avec la divinité. Dans ce contexte est significatif le fait qu'Érasme mette en œuvre un second concept, le genius. Or, le mot ne renvoie pas à l'image divine rattachée à l'idée d'inspiration impersonnelle, dans une doctrine qui, depuis Platon, définissait le poète comme instrument dépersonnalisé de la divinité. Chomarat nous rappelle que le genius roman fournit une image opposée de la divinité : il est le démon personnel de chaque pater familias ou des individus exceptionnels, qu'il accompagne tout au long de leur vie, dans leurs actes les plus quotidiens. Selon le même auteur, le mot "ajoute un rayonnement ou un halo à ce que disent plus simplement ingenium ou natura". ${ }^{53}$ Mais il est possible qu'Érasme s'intéresse aussi spécifiquement à la solidarité que le mot est censé exprimer entre l'individu humain et une hypostase quotidienne, personnalisée, de la divinité. Ce serait pour exprimer la personnalité unique, l'individualité saisie au nœud de son existence, qu'Érasme a besoin d'un nouveau mot, dans une volonté de se différencier nettement d'avec la fureur impersonnelle d'origine platonicienne. ${ }^{54}$

Il semblerait qu'avec le genius, un facteur de différenciation d'avec le style inspiré est mis en scène: la sacralité de la parole passe par la différence personnelle. Le genius, terme plus solidaire à la nature individuelle, est convoqué ici pour renforcer le sens de l'ingenium ${ }^{55}$ : il est mis au service

52 Cf. aussi Adag. II 5,44.

53 J. Chomarat, Grammaire et rhétorique ...., vol II, p. 833 sqq. Le mot genius est beaucoup moins présent dans le Ciceronianus que l'ingenium, autour duquel se concentre la conception qu'Érasme se fait de l'écrivain.

54 Lecointe fait remarquer l'attitude d'Érasme qui est en cela radicalement différent de celle du néoplatonisme : il utilise l'expression 'inspiration divine' dans des contextes humoristiques, sauf les cas où elle nomme le Saint-Esprit au sens rigoureusement religieux. La conclusion de Lecointe est que "Érasme ne prend pas le néoplatonisme au sérieux. Sa conception même de l'action du Saint-Esprit ... invite à l'approcher asymptomatiquement de celle du 'naturel', du moi profond de l'individu." (p. 435)

55 Il me semble que Lecointe n'arrive pas à expliquer le passage d'ingenium d'Érasme au génie du français moderne. Il fait seulement remarquer la préférence du néoplatonisme 
d'une nouvelle conception de la rhétorique, qui tire les dernières conséquences de la découverte chrétienne de l'intériorité. En assimilant cette dimension, l'ingenium devient un instrument adapté à la réflexion et à la caractérisation de l'individualité.

L'ingenium se définit indirectement tout au long du dialogue. Dans un premier moment, il est une limite indépassable dans la formation individuelle, qu'Érasme met en exergue sans cesse. ${ }^{56}$ On est loin de la nature de Quintilien, matière pour l'enseignement où on ne devrait pas rejeter ni même les natures médiocres. Pour Érasme, la nature ne se laisse pas modeler, violer ; l'art, tout en reconnaissant son importance, dépend d'une manière décisive de la nature. On peut seulement ajouter la nature à actualiser ses possibilités, mais on ne pourrait jamais la transformer dans une autre, ni aller à l'encontre de ses composantes. C'est pour cela que l'imitation éclectique est 'raisonnable' :

pourvu que tu te contentes d'imiter en Cicéron ce qu'il a de meilleur, que tu t'efforces à rivaliser avec ton modèle plutôt qu'à le suivre, que tu cherches à devenir son égal plutôt que son calque, et sans contrarier ton génie naturel ; en un mot, que tu n'essaies pas d'accorder ton discours à l'exemple de Cicéron plutôt qu'au sujet traité." (p. $322)^{57}$

S'il n'est pas possible d'imiter en tout le modèle, il possible - en revanche - de le dépasser. Le processus par lequel Buléphore conduit Nosopon vers la vraie idée d'éloquence repousse l'imitation servile, découvre l'imitation éclectique, pour aboutir finalement à l'émulation, la maxime liberté imaginable dans cette tradition. Il y a donc une liberté de l'ingenium qui s'affirme dans le choix complexe par lequel se forme un style personnel. Dans l'Ecclesiastes Érasme va encore plus loin dans cette direction, en conseillant au prédicateur de varier son sermon et en lui mettant aussi à disposition une longue liste des styles. Celle-ci est une liste ouverte qui

pour l'ingenium, mais sans relever sa présence dominante chez Érasme et la signification d'une deuxième notion qui lui est associée.

56 Chomarat fait remarquer ce que les différents mots de la famille ont en commun : "Avec ou sans coloration surnaturelle tous ces mots de la famille de nasci expriment la même idée, à la fois négative (limites de l'éducation) et positive (aptitudes innées).” (p. 834).

57 "modo, qua est optimus, hac aemuleris, modo aemuleris potius quam sequaris, modo studeas aequalis esse verius quam similis, modo ne pugnes adversus genium tuum, modo sic ne affectes congruere Ciceroni tuam orationem, ut rei de qua loqueris non congruat." (p. 214) 
comprend, à côté des trois styles traditionnels, une multitude de styles secondaires possibles ${ }^{58}$. L'Ecclesiastes semble ainsi tirer les dernières conséquences de la variété des styles individuels, thème débattu dans le Ciceronianus. Le système hiérarchique des styles éclate, la liste des styles possibles reste ouverte, car il existe une plasticité de la nature individuelle la rendant susceptible d'un enrichissement constant de ses formes d'expressions.

Mais c'est surtout dans l'analyse des styles individuels qu'on trouve la caractérisation de l'ingenium: traduit par 'génie', 'talent' 'dispositions naturelles' ou même 'intelligence', le terme est mis en œuvre pour dresser le profil spécifique de chacun des écrivains invoqués. Le mot est utilisé parfois pour désigner globalement un individu, sa nature ou sa personnalité ${ }^{59}$, mais le plus souvent on le trouve comme synonyme de talent, de dispositions naturelles associées à une pratique littéraire d'une diversité extrême. Dans cette acception le terme accepte plusieurs degrés : quelques écrivains ont un certain 'don naturel ${ }^{160}$, un certain talent ${ }^{61}$, d'autres 'un talent aussi heureux' ou même un 'talent divin'. L'ingenium intéresse tout le domaine de l'écriture, non seulement la création littéraire, la poésie, mais aussi les 'sciences' : Saint Jérôme a un 'génie supérieur tant par son éloquence que par sa science', et Jean Pic de la Mirandole est 'un génie vraiment divin, également capable de briller dans toutes les disciplines'. ${ }^{62}$

Le terme offre le nœud de la caractérisation individuelle, c'est autour de lui que les autres traits viennent s'aligner: l'ingenium a partie liée avec l'érudition, la force de l'éloquence, les mœurs et la déontologie, le jugement pénétrant, l'imagination forte etc. Tous ces éléments s'intègrent dans une image de l'individu qui est désormais celle de la persona. Véhicule de la réflexion sur la personnalité littéraire dans la culture antérieure, l'ingenium

58 'Et à part les trois 'caractères' oratoires que mentionnent les rhéteurs, il y a encore une foule d'autres différences. Il y a le style vif, le style ardent, le style apaisé ; il y a l'austère, le fleuri, le doux et l'amer, le travaillé et le simple ; il y a le transparent et le contourné, le direct et l'oblique ; il y a le diffus et le condensé." Ecclesiastes, in Omnia opera Desiderii Erasmi quaecumque ipse autor pro suis agnovit, Bâle, 1540, t. V, p. 843, fragment cité et traduit par Lecointe, p. 463.

59 p. 296, p. 351.

60 p. 329, p. 332.

${ }_{61}$ p. $334,335$.

62 p. 327. 
commence à être concurrencé par ce nouveau concept, destiné lui-même à une riche histoire à travers l'époque moderne.

L'élément le plus important de l'ingenium est défini sur la toile de fond de l'imitation éclectique : il s'agit de la capacité à créer de la nouveauté à partir de l'héritage devenu diffus des classiques. Contrairement à un courant que préconisait l'imitation dans l'acte même de l'écriture, pour Érasme l'imitation est seulement un point de départ, elle est effective dans la formation d'un ingenium, mais non plus dans le geste de la création :

Alors le talent naturel enrichi de ces nourritures variées crée de lui même un discours qui ne dégagera pas le parfum de telle fleur, de telle feuille ou de telle herbe, mais qui exprimera les dispositions naturelles et les sentiments du cœur" (p. 317)

La conception d'un ingenium individuel qui crée de lui même un discours franchit en quelque sorte les limites de la doctrine de l'imitation ${ }^{63}$. Les modèles ont une fonction formatrice, mais ils doivent s'effacer dans l'acte de création même, où il ne reste que l'ingenium, les dispositions naturelles et les sentiments, c'est-à-dire des aspects purement particuliers d'un esprit.

L'attitude de Nosopon se base sur la formalisation d'un style individuel : pour lui, le style idéal est l'expression d'une Idée universelle, qui a été objectivée une fois pour toutes dans une œuvre déterminée, celle de Cicéron. C'est pour cela que la reproduction fidèle des caractéristiques stylistiques de celle-ci est censée représenter l'expression d'un aboutissement personnel, et le critère d'excellence de toute nouvelle œuvre. Cette perspective aborde le style comme une activité de simple individuation: les traits stylistiques de l'œuvre modèle expriment une instance universelle. Il y a un rapport de parfaite coïncidence entre l'instance et les traits stylistiques du discours, car le style est parfaitement transparent, par conséquent, imitable, c'est-à-dire dépourvu de toute dimension strictement individuelle. Au contraire, pour Érasme le style exprime une individualité unique, qui ne saurait s'identifier à l'expression objective de l'universel, même s'il possède un caractère objectif issu de la relation entre style et réalité (la réalité de l'individu et de son

${ }^{63}$ À la fin du siècle, dans les traités en vernaculaire sur l'ingenium, la position antiimitative est déjà constituée : on le peut voir, par exemple, chez Huarte de San Juan, qui fait l'éloge de l'ingenium en tant que pouvoir créateur de l'esprit humain, et critique maintes fois les grands esprits de l'Antiquité, comme Platon, Aristote ou Hippocrate. (Huarte de San Juan, Examen de ingenios, ed. Guillermo Serés, Madrid, Cátedra, 1994) 
époque). Traduit dans la notion de aptum ou de decorum, ce caractère réel et adapté rend compte de la diversité des instances historiques susceptibles à s'exprimer à travers une pluralité des styles différents. La notion même du style a changé: il s'agit d'un style de l'individualité, qui marque une rupture radicale avec la conception traditionnelle de l'écriture, tout en conservant son cadre rhétorique.

Avec le Ciceronianus, la problématique de l'ingenium semble enregistrer un infléchissement important: pour rendre compte de l'individualité littéraire, il faut chercher dans son style, c'est-à-dire, dans son œuvre. L'œuvre est le lieu de manifestation de la personnalité. Les conséquences de ce changement pour le thème qui nous occupe sont très importantes, car il permet de poser dans une nouvelle forme le problème de l'individualité, de chercher plus profondément le profil de l'ingenium. C'est dans des traits stylistiques d'une œuvre que l'on essaie de saisir l'individualité, c'est à partir du style concret de l'œuvre que l'on essaie d'aboutir à une perspective théorique sur l'individualité en tant que facteur responsable de la création. Même si l'examen porte encore sur la personne de l'écrivain, sur ses qualités et ses défauts, il y a une nouvelle perspective sur l'œuvre en soi, devenue l'objet d'un regard objectivant.

Ce qui semble définir le dialogue c'est l'effort d'infléchir la caractérisation du style dans une perspective d'individualisation qui s'oppose manifestement à celle qui fait du style une activité de simple individuation. Nosopon pense que devenir écrivain est un processus d'individuation, il cherche à produire une œuvre selon des traits uniformes, dans un univers littéraire où l'écrivain ne peut être que cicéronien. L'œuvre cicéronienne fournit non seulement les idées, mais aussi, et d'une façon extrêmement réductrice, tout le matériau linguistique d'une nouvelle œuvre. Elle est le principe d'individuation pour tout le genre des œuvres des cicéroniens, le principe nécessaire pour identifier un auteur et son œuvre en tant que cicéroniens, et nullement en tant que 'bons', 'divins' etc. Dans cette perspective, il y aurait individuation, si autant l'auteur que l'œuvre peuvent prendre forme, se réaliser: cette œuvre de Nosopon sera distincte de cette autre d'un cicéronien ; même si elles se bâtissent avec des expressions identiques, leur combinatoire sera nécessairement différente. Mais en réalité, la pratique stylistique dépend essentiellement du procès d'individualisation, qui se 
produit dans la pratique stylistique, lorsque les problèmes d'identification font place à des problèmes de caractérisation. ${ }^{64}$ L'individu (l'ingenium, l'écrivain, en occurrence) est saisi selon des traits de distinction et de ressemblance par rapport aux autres de son genre ; c'est-à-dire, l'accent est alors mis sur des éléments d'intension du concept, non plus sur son extension. Dans le dialogue érasmien, Nosopon se situe dans une perspective de simple individuation, d'identification (est-ce l'écrivain $\mathrm{X}$ un cicéronien?) Mais l'examen des auteurs découvre une espèce à un seul individu, ce qui annule la signification du geste initial, qui assignait aux cicéroniens possibles la condition absolue d'écrivain. En revanche, dans l'analyse des talents individuels, Buléphore impose une perspective d'individualisation: les caractéristiques des écrivains invoqués se multiplient sans arrêt, dans une prolifération extrême des traits particuliers dont la limite est impossible à figer.

Il s'agit d'une nouvelle perspective sur le style, certes, mais elle semble signifier plus qu'un ajout à la perspective antérieure. En effet, le cicéronien Nosopon n'arrive pas à produire une œuvre concrète, car il a besoin de plusieurs semaines pour écrire une lettre. Il est malade, et non seulement de 'cicéronianisme', mais frappé aussi d'une impossibilité réelle d'écrire, ce qui semble nous indiquer que, dans la pratique stylistique, l'individuation est liée a l'individualisation. Toute œuvre se présente alors comme le résultat de ces deux activités, et toutes les deux se produisent dans le style. Le style, qui est le résultat de ces deux moments semble ainsi fonctionner comme lieu d'objectivation, de réalisation et de rencontre entre l'œuvre et l'auteur.

Le Ciceronianus constitue le nécessaire épilogue à une histoire où le profil de l'ingenium était encore diffus. Mais il fournit aussi l'articulation la plus nette d'une problématique que la modernité mettra en exergue. Le concept d'ingenium d'Érasme participe d'un univers rhétorique, où une signification esthétique plus particularisée commence à s'imposer, qui essaie de rendre compte de la dimension individuelle dans la pratique littéraire. Mais il témoigne aussi d'une autre tension de l'époque: est-ce possible de conceptualiser la création littéraire, l'individualité du créateur sur le fond d'une discipline globale qu'est la rhétorique ? La croissante nécessité d'exprimer

64 Vincent Descombes, Proust. Philosophie du roman, Paris, 1987, p. 301. 
l'individualité va à l'encontre de la perspective de simple individuation, perspective d'une rhétorique qui veut encore dominer l'ensemble de la culture, et empêcher tout mouvement de spécialisation. Cette tension qui informe le concept signale la présence d'un besoin moderne: on pourrait voir dans la spécialisation des disciplines une hypostase extrême du respect pour la différence individuelle, une dernière conséquence de la variété des ingenia, dont la reconnaissance s'esquisse d'abord dans l'univers du créateur, le plus caractérisé, mais aussi le plus marginalisé par la tradition antérieure. 